\title{
EXACT LEFSCHETZ FIBRATIONS ASSOCIATED WITH DIMER MODELS
}

\author{
Masahiro Futaki and Kazushi Ueda
}

\begin{abstract}
We associate an exact Lefschetz fibration with a pair of a consistent dimer model and an internal perfect matching on it, whose Fukaya category is derived equivalent to the category of representations of the directed quiver with relations associated with the pair. As a corollary, we obtain a version of homological mirror symmetry for two-dimensional toric Fano stacks.
\end{abstract}

\section{Introduction}

A dimer model is a bicolored graph on a 2-torus $T=\mathbb{R}^{2} / \mathbb{Z}^{2}$ encoding the information of a quiver with relations. It is originally introduced by string theorists (see e.g. a review by Kennaway [16] and references therein) and studied by mathematicians from various points of view. If a dimer model $G$ is consistent, then the quiver $\Gamma$ with relations associated with $G$ has the following properties:

- The derived category $D^{b} \bmod _{0} \mathbb{C} \Gamma$ of finitely-generated nilpotent representations of $\Gamma$ is Calabi-Yau in the sense that the Serre functor is a shift functor ([24, Theorem 6.3], see also [3, 5]).

- The moduli space $\mathcal{M}_{\theta}$ of stable representations of $\Gamma$ with dimension vector $(1, \ldots, 1)$ and a generic stability parameter $\theta$ is a smooth toric Calabi-Yau 3 -fold [11, Theorem 6.4].

- There is an equivalence

$$
D^{b} \operatorname{coh} \mathcal{M}_{\theta} \cong D^{b} \bmod \mathbb{C} \Gamma
$$

of triangulated categories between the derived category of coherent sheaves on $\mathcal{M}_{\theta}$ and the derived category of finitely generated $\mathbb{C} \Gamma$-modules ([10, Theorem $14.1]$, see also $[2,33])$. This restricts to an equivalence

$$
D^{b} \operatorname{coh}_{0} \mathcal{M}_{\theta} \cong D^{b} \bmod _{0} \mathbb{C} \Gamma
$$

where $D^{b} \operatorname{coh}_{0} \mathcal{M}_{\theta}$ is the derived category of coherent sheaves on $\mathcal{M}_{\theta}$ supported at the inverse image of the origin by the natural morphism $\mathcal{M}_{\theta} \rightarrow$ $\operatorname{Spec} \Gamma\left(\mathcal{O}_{\mathcal{M}_{\theta}}\right)$.

- A toric divisor on $\mathcal{M}_{\theta}$ corresponds to a perfect matching $D$ on $G$ [11, Lemma 6.1]. A perfect matching $D$ is said to be internal if it corresponds to a compact toric divisor of $\mathcal{M}_{\theta}$ for some stability parameter $\theta$. One can associate a directed subquiver $\Gamma^{\rightarrow}$ of $\Gamma$ with an internal perfect matching on $G$. 
- For any two-dimensional toric Fano stack $X$, there is a pair $(G, D)$ of a consistent dimer model $G$ and a perfect matching $D$ on $G$ such that there is an equivalence

$$
D^{b} \operatorname{coh} X \cong D^{b} \bmod \mathbb{C} \Gamma^{\rightarrow}
$$

of triangulated categories [9, Thoerem 1.1].

We prove the following in this paper:

Theorem 1.1. For a pair $(G, D)$ of a consistent dimer model $G$ and a perfect matching $D$ on $G$, there is an exact Lefschetz fibration $p: \mathcal{S} \rightarrow \mathbb{C}$ such that

$$
D^{b} \mathfrak{F u k} p \cong D^{b} \bmod \mathbb{C} \Gamma^{\rightarrow} .
$$

By combining Theorem 1.1 with (1), one obtains an equivalence

$$
D^{b} \mathfrak{F} \mathfrak{k} p \cong D^{b} \operatorname{coh} X
$$

of triangulated categories, which is a version of homological mirror symmetry for two-dimensional toric Fano stacks. Homological mirror symmetry is proposed by Kontsevich, originally for Calabi-Yau manifolds [18] and later generalized to Fano manifolds [19]. The definition of the Fukaya category of a Lefschetz fibration is due to Seidel [28, 30]. Homological mirror symmetry for toric stacks is started in [27] and studied by many people. The relation between dimer models and homological mirror symmetry is discovered in [6] and followed up in [32, 31].

Theorem 1.1 implies homological mirror symmetry for toric Calabi-Yau 3-folds just as in [26, Theorem 1.1]:

Corollary 1.2. For a smooth toric Calabi-Yau 3-fold $K$ with a compact toric divisor, there is an exact symplectic manifold $H$ and a full embedding of triangulated categories

$$
D^{b} \operatorname{coh}_{0} K \hookrightarrow D^{b} \mathfrak{F u k} H .
$$

The organization of this paper is as follows: In Section 2, we recall basic definitions on dimer models and $A_{\infty}$-categories, and introduce the $A_{\infty}$-category associated with a dimer model. In Section 3, we study the subcategory of $\mathcal{A}_{G}$ associated with an internal perfect matching, and discuss its relation with the derived category of modules over the path algebra. In Section 4, we construct an exact Lefschetz fibration from a dimer model and prove Theorem 1.1 and Corollary 1.2.

\section{An $A_{\infty}$-category from a dimer model}

We first recall basic definitions on dimer models:

- A dimer model is a bicolored graph $G=(B, W, E)$ on an oriented 2-torus $T=\mathbb{R}^{2} / \mathbb{Z}^{2}$ which divides $T$ into polygons. Here $B$ is the set of black nodes, $W$ is the set of white nodes, and $E$ is the set of edges. No edge is allowed to connect nodes with the same color.

- A quiver consists of a finite set $V$ called the set of vertices, another finite set $A$ called the set of arrows, and two maps $s, t: A \rightarrow V$ called the source and the target map. The quiver $Q=(V, A, s, t)$ associated with $G$ is defined as the dual graph of $G$, equipped with the orientation so that the white node is always on the right of an arrow; the set $V$ of vertices is the set of faces of $G$, 
and the set $A$ of arrows can naturally be identified with the set $E$ of edges of $G$.

- For a cyclic path $p=\left(a_{n}, \ldots, a_{1}\right)$ and an arrow $b$ on a quiver $Q$, the derivative of $p$ by $b$ is defined by

$$
\frac{\partial p}{\partial b}=\sum_{i=1}^{n} \delta_{a_{i}, b}\left(a_{i-1}, a_{i-2}, \ldots, a_{1}, a_{n}, a_{n-1}, \ldots, a_{i+1}\right),
$$

where

$$
\delta_{a, b}= \begin{cases}1 & a=b, \\ 0 & \text { otherwise. }\end{cases}
$$

A potential $\Phi$ is a linear combination of cyclic paths on the quiver. The derivative of $\Phi$ is defined by linearity, and the two-sided ideal $\mathcal{I}=(\partial \Phi)$ generated by the derivatives $\partial \Phi / \partial a$ for all arrows $a$ gives relations of the quiver.

- The potential $\Phi$ of the quiver $Q$ associated with a dimer model is defined by

$$
\Phi=\sum_{w \in W} c(w)-\sum_{b \in B} c(b),
$$

where $c(n)$ for $n \in B \sqcup W$ is the minimal cyclic path around $n$.

- A perfect matching is a subset $D \subset E$ such that for any $n \in B \sqcup W$, there is a unique edge $e \in D$ adjacent to $n$. A dimer model is non-degenerate if for any edge $e \in E$, there is a perfect matching $D$ such that $e \in D$.

- Two paths $p$ and $q$ are said to be weakly equivalent if $p r$ is equivalent to $q r$ for some other path $r$. A non-degenerate dimer model is said to be consistent if weakly equivalent paths are equivalent.

Next we recall the difinition of an $A_{\infty}$-category. For a $\mathbb{Z}$-graded vector space $N=\bigoplus_{j \in \mathbb{Z}} N^{j}$ and an integer $i$, the $i$-th shift of $N$ to the left will be denoted by $N[i]$; $(N[i])^{j}=N^{i+j}$. An $A_{\infty}$-category $\mathcal{A}$ consists of

- the set $\mathfrak{O} \mathfrak{b}(\mathcal{A})$ of objects,

- for $c_{1}, c_{2} \in \mathfrak{O b}(\mathcal{A})$, a $\mathbb{Z}$-graded vector space $\operatorname{hom}_{\mathcal{A}}\left(c_{1}, c_{2}\right)$ called the space of morphisms, and

- operations

$$
\mathfrak{m}_{l}: \operatorname{hom}_{\mathcal{A}}\left(c_{l-1}, c_{l}\right) \otimes \cdots \otimes \operatorname{hom}_{\mathcal{A}}\left(c_{0}, c_{1}\right) \longrightarrow \operatorname{hom}_{\mathcal{A}}\left(c_{0}, c_{l}\right)
$$

of degree $2-l$ for $l=1,2, \ldots$ and $c_{i} \in \mathfrak{O b}(\mathcal{A}), i=0, \ldots, l$, satisfying the $A_{\infty}$-relations

$$
\sum_{i=0}^{l-1} \sum_{j=i+1}^{l}(-1)^{\operatorname{deg} a_{1}+\cdots+\operatorname{deg} a_{i}-i} \mathfrak{m}_{l+i-j+1}\left(a_{l} \otimes \cdots \otimes a_{j+1} \otimes \mathfrak{m}_{j-i}\left(a_{j} \otimes \cdots \otimes a_{i+1}\right)\right.
$$

$$
\left.\otimes a_{i} \otimes \cdots \otimes a_{1}\right)=0
$$

for any positive integer $l$, any sequence $c_{0}, \ldots, c_{l}$ of objects of $\mathcal{A}$, and any sequence of morphisms $a_{i} \in \operatorname{hom}_{\mathcal{A}}\left(c_{i-1}, c_{i}\right)$ for $i=1, \ldots, l$.

A cyclic $A_{\infty}$-category of dimension $d \in \mathbb{Z}$ is a pair $(\mathcal{A},\langle\bullet, \bullet\rangle)$ of an $A_{\infty}$-category and a non-degenerate pairing

$$
\langle\bullet, \bullet\rangle: \operatorname{hom}\left(c_{2}, c_{1}\right) \otimes \operatorname{hom}\left(c_{1}, c_{2}\right) \rightarrow \mathbb{C}[d]
$$


which is both symmetric

$$
\langle x, y\rangle+(-1)^{(\operatorname{deg} x-1)(\operatorname{deg} y-1)}\langle y, x\rangle=0
$$

and cyclic

$$
\left\langle\mathfrak{m}_{n}\left(x_{n}, \ldots, x_{1}\right), x_{0}\right\rangle=(-1)^{\left(\operatorname{deg} x_{n}-1\right)\left(\operatorname{deg} x_{n-1}+\cdots+\operatorname{deg} x_{0}-n\right)}\left\langle\mathfrak{m}_{n-1}\left(x_{n-1}, \ldots, x_{0}\right), x_{n}\right\rangle .
$$

As shown in [20, Section 8.1], one can associate a cyclic $A_{\infty}$-category of dimension three with any quiver with potential. By applying their construction to the quiver with potential associated with a dimer model, one obtains the following cyclic $A_{\infty^{-}}$ category:

Definition 2.1. Let $G=(B, W, E)$ be a dimer model and $\Gamma=(V, A, s, t, \mathcal{I})$ be the quiver with relations associated with $G$. Then the $A_{\infty}$-category $\mathcal{A}$ associated with $G$ is defined as follows:

- The set of objects is the set $V$ of vertices of $\Gamma$.

- For two objects $v$ and $w$ in $\mathcal{A}$, the space of morphisms is given by

$$
\operatorname{hom}_{\mathcal{A}}^{i}(v, w)= \begin{cases}\mathbb{C} \cdot \operatorname{id}_{v} & i=0 \text { and } v=w, \\ \operatorname{span}\{a \mid a: w \rightarrow v\} & i=1, \\ \operatorname{span}\left\{a^{\vee} \mid a: v \rightarrow w\right\} & i=2, \\ \mathbb{C} \cdot \operatorname{id}_{v}^{\vee} & i=3 \text { and } v=w, \\ 0 & \text { otherwise. }\end{cases}
$$

- Non-zero $A_{\infty}$-operations are

$$
\mathfrak{m}_{2}^{\mathcal{A}}\left(x, \mathrm{id}_{v}\right)=\mathfrak{m}_{2}^{\mathcal{A}}\left(\mathrm{id}_{w}, x\right)=x
$$

for any $x \in \operatorname{hom}_{\mathcal{A}}(v, w)$,

$$
\mathfrak{m}_{2}^{\mathcal{A}}\left(a, a^{\vee}\right)=\mathrm{id}_{v}^{\vee}
$$

and

$$
\mathfrak{m}_{2}^{\mathcal{A}}\left(a^{\vee}, a\right)=\mathrm{id}_{w}^{\vee}
$$

for any arrow $a$ from $v$ to $w$,

$$
\mathfrak{m}_{k}^{\mathcal{A}}\left(a_{1}, \ldots, a_{k}\right)=a_{0} .
$$

for any cycle $\left(a_{0}, \ldots, a_{k}\right)$ of the quiver going around a white node, and

$$
\mathfrak{m}_{k}^{\mathcal{A}}\left(a_{1}, \ldots, a_{k}\right)=-a_{0} .
$$

for any cycle $\left(a_{0}, \ldots, a_{k}\right)$ of the quiver going around a black node.

- The pairing

$$
\langle\bullet, \bullet\rangle: \operatorname{hom}_{\mathcal{A}}(w, v) \otimes \operatorname{hom}_{\mathcal{A}}(v, w) \rightarrow \mathbb{C}[3]
$$

is defined by

and zero otherwise.

$$
\left\langle a^{\vee}, a\right\rangle=\left\langle\mathrm{id}_{v}^{\vee}, \mathrm{id}_{v}\right\rangle=1
$$

If a dimer model $G$ is consistent, then the derived category $D^{b} \mathcal{A}$ of the $A_{\infty}$-category $\mathcal{A}$ associated with $G$ is equivalent to the derived category $D^{b} \bmod _{0} \mathbb{C} \Gamma$ of nilpotent representations of $\Gamma$ : 
Proposition 2.2. For a dimer model $G$, let $\mathcal{A}$ be the cyclic $A_{\infty}$-category associated with $G$ and $\Gamma$ be the quiver with relations associated with $G$. If $G$ is consistent, then there is an equivalence

$$
D^{b} \mathcal{A} \cong D^{b} \bmod _{0} \mathbb{C} \Gamma
$$

of triangulated categories.

Sketch of proof. Let $\mathcal{C}$ be the full subcategory of the differential graded enhancement of $D^{b} \bmod \mathbb{C} \Gamma$ consisting of simple modules. Since $D^{b} \bmod _{0} \mathbb{C} \Gamma$ is the smallest triangulated subcategory of $D^{b} \bmod \mathbb{C} \Gamma$ containing simple modules, one has an equivalence

$$
D^{b} \mathcal{C} \cong D^{b} \bmod _{0} \mathbb{C} \Gamma
$$

by Bondal and Kapranov $[1, \S 4$, Theorem 1]. Hence the equivalence (3) follows from the quasi-equivalence

$$
\mathcal{C} \cong \mathcal{A}
$$

of $A_{\infty}$-categories, which can be shown directly using homological perturbation theory $[12,8,23,21]$, or deduced from the case of a directed subcategory discussed in Section 3 by noting that $\mathcal{A}$ is the trivial extension of its directed subcategory [4].

An alternative approach, suggested to the authors by Bernhard Keller, is to use Koszul duality for $A_{\infty}$-categories developed by Lefèvre-Hasegawa [22] and summarized in [13]: As $\mathcal{A}$ is augmented over the product of copies of $\mathbb{C}$ indexed by the vertices of $Q$, the bar construction

$$
C=B \mathcal{A}=\bigoplus_{n=0}^{\infty} \mathcal{A}^{\otimes n}
$$

equipped with the co-differential $\delta: C \rightarrow C$ defined by

$$
\begin{aligned}
\delta\left(a_{l} \otimes \cdots \otimes a_{1}\right)= & \sum_{i=0}^{l-1} \sum_{j=i+1}^{l}(-1)^{\operatorname{deg} a_{1}+\cdots+\operatorname{deg} a_{i}-i} a_{l} \otimes \cdots \otimes a_{j+1} \\
& \otimes \mathfrak{m}_{j-i}\left(a_{j} \otimes \cdots \otimes a_{i+1}\right) \otimes a_{i} \otimes \cdots \otimes a_{1},
\end{aligned}
$$

is a co-augmented differential graded coalgebra, and each $A_{\infty}$-module $\mathcal{M}$ over $\mathcal{A}$ yields a differential graded comodule

$$
B \mathcal{M}=\bigoplus_{n=0}^{\infty} \mathcal{M} \otimes \mathcal{A}^{\otimes n}
$$

over $C$. The functor $\mathcal{M} \mapsto B \mathcal{M}$ induces an equivalence

$$
D^{b} \mathcal{A} \rightarrow D_{0} C
$$

from the bounded derived category of $\mathcal{A}$ to the full triangulated subcategory $D_{0} C$ of the coderived category $D C$ of $C$ generated by differential graded comodules coming from the co-augmentation.

Now let $G$ be the completion of the differential graded algebra associated with the quiver $(Q, \Phi)$ with potential by Ginzburg [7, Section 1.4]. Then $G$ is the $\mathbb{C}$ dual of $C$ as observed in [15, Section 5.3], and $\mathbb{C}$-duality transforms each differential graded $C$-comodule into a differential graded $G$-module. This induces a contravariant equivalence

$$
\operatorname{Hom}_{\mathbb{C}}(\bullet, \mathbb{C}): D_{0} C \rightarrow D_{0}(G)
$$


from $D_{0} C$ to the full triangulated subcategory $D_{0}(G)$ of the derived category $D(G)$ of $G$ containing simple modules. If the dimer model is consistent, then $\mathbb{C} \Gamma$ is a CalabiYau 3 algebra and one has a quasi-isomorphism $\mathbb{C} \Gamma \stackrel{\sim}{\rightarrow} G$ by [7, Theorem 5.3.1], so that $D_{0}(G)$ is equivalent to $D^{b} \bmod _{0} \mathbb{C} \Gamma$. One can obtain a covariant equivalence instead of a contravariant one by composing with the duality functor $\mathbb{R} \operatorname{Hom}_{G}(\bullet, G)$ : $D_{0}(G) \rightarrow D_{0}(G)$.

\section{The directed subcategory}

Let $G=(B, W, E)$ be a dimer model and $Q=(V, A, s, t)$ be the quiver associated with $G$. A perfect matching $D$ defines a subquiver

$$
Q_{D}=\left(V, A_{D}, s_{D}, t_{D}\right)
$$

of $Q$ with the same set of vertices as $Q$ and the set

$$
A_{D}=A \backslash D
$$

of arrows consisting of those not in $D \subset E=A$. The path algebra $\mathbb{C} Q_{D}$ is naturally a subalgebra of $\mathbb{C} Q$, and the intersection $I_{D}=\mathbb{C} Q_{D} \cap \mathcal{I}$ of the ideal $\mathcal{I}$ of relations on $Q$ with $\mathbb{C} Q_{D}$ gives an ideal of relations on $Q_{D}$. We write the resulting quiver with relations as $\Gamma_{D}=\left(Q_{D}, \mathcal{I}_{D}\right)$.

Let $\mathcal{M}_{\theta}$ be the moduli space of stable representations of $\Gamma$ with respect to a stability parameter $\theta$ in the sense of King [17]. If $G$ is non-degenerate, then $\mathcal{M}_{\theta}$ is a smooth toric variety for a generic $\theta$, and for any toric divisor in $\mathcal{M}_{\theta}$, there is a perfect matching $D$ such that the divisor is defined as the zero locus of the arrows dual to edges in $D$ [11]. In addition, for any perfect matching $D$, there is a generic stability parameter $\theta$ such that $D$ corresponds to a toric divisor in $\mathcal{M}_{\theta}$ in this way.

A perfect matching $D$ is said to be internal if the toric divisor in $\mathcal{M}_{\theta}$ corresponding to $D$ for some $\theta$ is compact. It is easy to see that a perfect matching $D$ is internal if and only if $Q_{D}$ does not have an oriented cycle.

For a total order $>$ on the set $V$ of vertices of $Q$, the directed subquiver $Q \rightarrow$ is defined as the subquiver of $Q$ whose set of vertices is the same as $Q$ and whose set of arrows $A \rightarrow$ is given by

$$
A^{\rightarrow}=\{a \in A \mid s(a)<t(a)\} .
$$

The subquiver $Q \rightarrow$ equipped with the relations $\mathcal{I}^{\rightarrow}=\mathbb{C} Q \rightarrow \cap \mathcal{I}$ will be denoted by $\Gamma \rightarrow$. A perfect matching $D$ is said to come from a total order $>$ if $\Gamma_{D}=\Gamma^{\rightarrow}$.

Lemma 3.1. A perfect matching $D$ of a dimer model $G$ is internal if and only if it comes from a total order $<$ on the set of faces of $G$.

Proof. It is clear that a perfect matching coming from a total order on the set of faces is internal. To show the converse, assume that a perfect matching $D$ is internal. The non-existence of oriented cycles ensures that this quiver defines a partial order on the set of vertices of the quiver. Choose any total order $<$ compatible with this partial order. Then the condition that $D$ is a perfect matching implies that the arrows contained in $D$ is precisely the arrows $a$ such that $s(a)>t(a)$; the path $p(a)$ from $t(a)$ to $s(a)$ which goes around either node adjacent to the edge dual to $a$ is contained in the subquiver and induces the order $s(a)>t(a)$. 
For an $A_{\infty}$-category $\mathcal{A}$ and a sequence $\boldsymbol{Y}=\left(Y_{1}, \ldots, Y_{m}\right)$ of objects, the associated directed subcategory $\mathcal{A}^{\rightarrow}=\mathcal{A}(\boldsymbol{Y})$ is the $A_{\infty}$-subcategory consisting of $\boldsymbol{Y}$ such that the spaces of morphisms are given by

$$
\operatorname{hom}_{\mathcal{A} \rightarrow}\left(Y_{i}, Y_{j}\right)= \begin{cases}\mathbb{C} \cdot \operatorname{id}_{Y_{i}} & i=j, \\ \operatorname{hom}_{\mathcal{A}}\left(Y_{i}, Y_{j}\right) & i<j \\ 0 & \text { otherwise }\end{cases}
$$

with the $A_{\infty}$-operations inherited from $\mathcal{A}$. If $\mathcal{A}$ comes from a dimer model $G$ as in Section 2 and $<$ is a total order on the set of vertices of the quiver associated with $G$, then we set $\boldsymbol{Y}=\left(Y_{1}, \ldots, Y_{m}\right)$ to be the set of objects of $\mathcal{A}$, arranged in the order inverse to the one induced by $<$.

Proposition 3.2. Let $G$ be a consistent dimer model and $>$ be a total order on the set of faces of $G$ giving an internal perfect matching. Then one has an equivalence

$$
D^{b} \mathcal{A}^{\rightarrow} \cong D^{b} \bmod \mathbb{C} \Gamma^{\rightarrow}
$$

of triangulated categories.

Proposition 3.2 comes from a quasi-equivalence $\mathcal{A}^{\rightarrow} \cong \mathcal{C}^{\rightarrow}$ with the the full subcategory $\mathcal{C} \rightarrow$ of the differential graded enhancement of $D^{b} \bmod \mathbb{C} \Gamma \rightarrow$ consisting of simple modules. This is an easy exercise in homological perturbation theory, and can also be deduced from [14, Proposition 2] as discussed in [4].

\section{An exact Lefschetz fibration from a dimer model}

We prove the following in this section, which together with Proposition 3.2 immediately implies Theorem 1.1:

Theorem 4.1. Let $G$ be a consistent dimer model and $D$ be an internal perfect matching on $G$. Then there is an exact Lefschetz fibration $p: X \rightarrow \mathbb{C}$ whose Fukaya category is equivalent to the directed $A_{\infty}$-category $\mathcal{A}^{\rightarrow}$ associated with $(G, D)$.

An exact Lefschetz fibration is a $J$-holomorphic function $p: \mathcal{S} \rightarrow \mathbb{C}$ on an exact almost Kähler manifold $(\mathcal{S}, \omega, J)$ such that all the critical points are non-degenerate. We also assume that $J$ is integrable near the critical points, and the horizontal lift $\widetilde{\gamma}_{x}$ : $[0,1] \rightarrow X$ of a smooth path $\gamma:[0,1] \rightarrow \mathbb{C}$ starting at $x \in p^{-1}(\gamma(0))$ is always defined. A distinguished basis of vanishing cycles is a collection $\left(C_{1}, \ldots, C_{m}\right)$ of Lagrangian spheres in the regular fiber of $p$ which collapse to critical points by parallel transport along a distinguished set of vanishing paths, cf. [30, Section 16]. By the Fukaya category of $p$, we mean the directed subcategory of the Fukaya category of the regular fiber of $p$ consisting of a distinguished basis of vanishing cycles. Recall from [30, Lemma 16.9] that any collection of framed exact Lagrangian spheres in an exact symplectic manifold can be realized as a distinguished basis of vanishing cycles of an exact Lefschetz fibration.

Lemma 4.2. There is a 2-manifold $M$ and a collection $\left(C_{v}\right)_{v \in V}$ of embedded circles on $M$ such that intersections of $C_{v}$ and $C_{v^{\prime}}$ are transverse and in natural bijection with arrows between $v$ and $v^{\prime}$. 


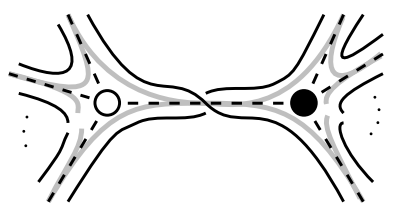

Figure 1. The surface and immersed circles associated with a dimer model

Proof. Recall that a ribbon graph is a graph together with the choice of a cyclic order on the set of edges connected to each node. A graph underlying a dimer model naturally has a ribbon structure by first giving the cyclic order on the set of edges around each node coming from the orientation of $T$, and then reversing those around the black nodes. A ribbon graph determines a 2-manifold $M$ by assigning a disk to each node and gluing them together as designated by the ribbon structure.

This construction can be described pictorially as follows: One first associates a disk with each node of the dimer model, and reverse the orientation of the disks asosciated with black nodes. Then the surface is obtained by connecting adjacent disks with a "twisted strip", so that the dimer model one has started with is naturally embedded on the surface (and in fact a deformation retraction of it). Figure 1 shows a part of a dimer model and the resulting surface near an edge.

For each vertex $v$ of $\Gamma$ (i.e. for each face of $G$ ), one can associate an immersed circle $C_{v}$ in $M$ as shown in light gray in Figure 1, so that arrows of $\Gamma$ naturally correspond to intersection points between them. They do not have self-intersections since the quiver $\Gamma$ does not have a loop, i.e. an arrow $a$ such that $s(a)=t(a)$; if such an arrow exists, no perfect matching comes from a total order on the set of vertices of $\Gamma$, so that there can be no internal perfect matching by Lemma 3.1.

We let $M$ and $C_{v}$ denote the 2-manifold and the embedded circles constructed in the proof of Lemma 4.2 henceforth.

Lemma 4.3. The 2-manifold $M$ admits an exact symplectic form $\omega$ such that $C_{v}$ are exact Lagrangian submanifolds.

Proof. Choose an exact complete Kähler structure on $M$ and let $\theta$ be a one-form on $M$ such that $\omega=d \theta$ is the Kähler form. Recall that $C_{v}$ is said to be exact if $\int_{C_{v}} \theta=0$. If we perturb $C_{v}$ to $C_{v}^{\prime}$, then Stokes' theorem states that

$$
\int_{C_{v}} \theta-\int_{C_{v}^{\prime}} \theta=\int_{D} \omega
$$

where $D$ is the region surrounded by $C_{v}$ and $C_{v}^{\prime} ; \partial D=C_{v}-C_{v}^{\prime}$. Note that both sides of $C_{v}$ contains a point at infinity, i.e., a point in $\bar{M} \backslash M$ where $\bar{M}$ is a compactification of $M$. It follows that for any $R \in \mathbb{R}$, one can choose $C_{v}^{\prime}$ such that $\int_{D} \omega=R$. By choosing $R=\int_{C_{v}} \theta$, one obtains $\int_{C_{v}^{\prime}} \theta=0$ as desired.

Let

$$
p: \mathcal{S} \rightarrow \mathbb{C}
$$

be an exact Lefschetz fibration such that $p^{-1}(0) \cong M$ and $\left(C_{v}\right)_{v \in V}$ forms a distinguished basis of vanishing cycles. To equip the Fukaya category with a $\mathbb{Z}$-grading, we need gradings of $M$ and $C_{v}$ : A grading of a symplectic manifold $(M, \omega)$ is the 
choice of a fiberwise universal cover $\widetilde{\mathcal{L a g}}_{M}$ of the Lagrangian Grassmannian bundle ${\mathcal{L} a g_{M}}_{M} M$. A Lagrangian submanifold $L \subset M$ naturally gives a section

$$
\begin{array}{cccc}
s_{L}: \quad L & \rightarrow & \left.\operatorname{Lag}_{M}\right|_{L} \\
\Psi & & \Psi \\
x & \mapsto & T_{x} L,
\end{array}
$$

and a grading of $L$ is a choice of a lift of $s_{L}$ to

$$
\widetilde{s}_{L}:\left.L \rightarrow \widetilde{\mathcal{L} a g}_{M}\right|_{L}
$$

When $M$ is equipped with a compatible almost complex structure so that $(M, \omega, J)$ is an almost Kähler manifold, the choice of a grading of $M$ is equivalent to the choice of a nowhere-vanishing continuous section $\eta$ of the square $\left(\Lambda^{\mathrm{top}} T^{*} M\right)^{\otimes 2}$ of the canonical bundle. Such a section exists if and only if $2 c_{1}(T M, J)=0 \in H^{2}(M, \mathbb{Z})$, and the homotopy classes of sections are classified by $H^{1}(M, \mathbb{Z})$. A section $\eta$ induces a map

$$
\begin{array}{rlrl}
\operatorname{det}_{\eta}^{2}: & \mathcal{L a g}_{M} & \rightarrow & \mathbb{C}^{\times} / \mathbb{R}^{>0} \cong S^{1} \\
\Psi & & \\
\operatorname{span}\left\{e_{1}, \ldots, e_{n}\right\} & \mapsto & {\left[\eta\left(\left(e_{1} \wedge \cdots \wedge e_{n}\right)^{\otimes 2}\right)\right] .}
\end{array}
$$

The composition of $s_{L}$ and $\operatorname{det}_{\eta}^{2}$ will be denoted by $\phi_{L}$, and a grading of $L$ is equivalent to a lift $\widetilde{\phi}_{L}: L \rightarrow \mathbb{R}$ of $\phi_{L}: L \rightarrow S^{1}$ to the universal cover $\mathbb{R} \rightarrow S^{1}$.

Given a pair $\left(L_{1}, L_{2}\right)$ of graded Lagrangian submanifolds, one can define the Maslov index $\mu\left(x ; L_{1}, L_{2}\right)$ for each intersection point $x \in L_{1} \cap L_{2}$. If $\operatorname{dim}_{\mathbb{C}} M=1$, then it is given by the round-up

$$
\mu\left(x ; L_{1}, L_{2}\right)=\left\lfloor\widetilde{\phi}_{L_{2}}(x)-\widetilde{\phi}_{L_{1}}(x)\right\rfloor
$$

of the difference of the phase function at $x$.

Lemma 4.4. There is a grading of $(M, \omega)$ and $C_{v}$ such that the Maslov index of $e \in C_{v} \cap C_{w}$ for $v>w$ is given by

$$
\mu\left(e ; C_{v}, C_{w}\right)= \begin{cases}1 & e \notin D \\ 2 & e \in D .\end{cases}
$$

Proof. Since $\operatorname{dim}_{\mathbb{R}} M=2$ in our case, the Lagrangian Grassmannian bundle is the principal $S^{1}$-bundle associated with $(T M)^{\otimes 2}$, and a grading is a trivialization (i.e., a section) of it. Let $\widetilde{G}$ be the pull-back of $G$ on $T=\mathbb{R}^{2} / \mathbb{Z}^{2}$ to the universal cover $\mathbb{R}^{2}$ of $T$, and $\widetilde{M}$ be the 2 -manifold associated with $\widetilde{G}$. Fix a vertex $\tilde{v}_{0}$ of the quiver associated with $\widetilde{G}$ and choose a grading of a tubular neighborhood of $C_{\tilde{v}_{0}} \subset \widetilde{M}$ so that $C_{\tilde{v}_{0}}$ admits a grading. Fix a grading of $C_{\tilde{v}_{0}}$ and choose a grading of $C_{\tilde{v}}$ for other $\tilde{v}$ successively as follows; if a vertex $\tilde{v}$ is adjacent by an arrow $a$ to another vertex $\tilde{v}^{\prime}$ where the gradings of a tubular neighborhood of $C_{\tilde{v}^{\prime}}$ and of $C_{\tilde{v}^{\prime}}$ are already defined, we choose a grading of a tubular neighborhood of $C_{\tilde{v}}$ and of $C_{\tilde{v}}$ so that the Maslov index $\mu\left(a ; C_{\tilde{v}^{\prime}}, C_{\tilde{v}}\right)$ is one if $\tilde{v}^{\prime}>\tilde{v}$, and minus one if $\tilde{v}^{\prime}<\tilde{v}$. Since these gradings on the tubular neighborhoods of vanishing cycles glue together coherently around a node of $\widetilde{G}$, this defines gradings of $\widetilde{M}$ and $C_{\tilde{v}}$, which descends to gradings of $M$ and $C_{v}$. 

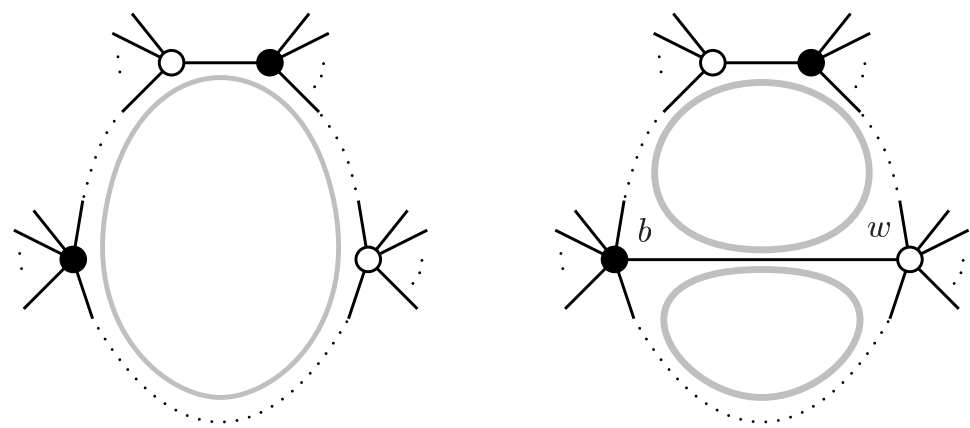

Figure 2. The passage from $G^{\prime}$ (left) to $G$ (right), where one vanishing cycle is divided into two

A relative grading $\eta$ of an exact Lefschetz fibration $p: \mathcal{S} \rightarrow \mathbb{C}$ is a nowhere-vanishing section of the holomorphic line bundle $\Lambda^{\text {top }}\left(T^{*} \mathcal{S}\right)^{\otimes 2} \otimes p^{*}\left(T^{*} \mathbb{C}\right)^{\otimes(-2)}$, which induces a grading on a regular fiber of $p$. One can equip $p$ with a relative grading such that the induced grading on $M$ coincides with the one in Lemma 4.4 [30, Section (16f)].

The following lemma concludes the proof of Theorem 4.1:

Lemma 4.5. Let $\left(e_{0}, e_{1}, \ldots, e_{k}\right)$ be the sequence of edges of $G$ around a node respecting the cyclic order such that $e_{0} \in D$. Then one has an $A_{\infty}$-operation

$$
\mathfrak{m}_{k}\left(e_{k}, \ldots, e_{1}\right)= \pm e_{0}
$$

in the Fukaya category $\mathfrak{F u k} p$ of Lefschetz fibration, where the sign is positive if the node is white, and negative if it is black.

Proof. Since $M$ is a 2-manifold, the $A_{\infty}$-operations in the Fukaya category are given by counting polygons bounded by Lagrangian submanifolds. It is clear from Figure 1 that each node of the dimer model gives such a polygon, and the non-existence of other polygons follows from the fact that the dimer model is a deformation retraction of the surface. This shows that $A_{\infty}$-operations are given as in (4) and only the sign is the issue here. We write the vanishing cycles surrounding a polygon as $C_{v_{i}}, i=0, \ldots, k$ so that $e_{0} \in C_{v_{0}} \cap C_{v_{k}}$ and $e_{i} \in C_{v_{i-1}} \cap C_{v_{i}}$ for $i=1, \ldots, k$. The grading of $C_{v_{i}}$ defines an orientation of $C_{v_{i}}$, and let $\xi_{i}, i=1, \ldots, k$ be the unit tangent vector of $C_{v_{i}}$ at $e_{i} \in C_{v_{i-1}} \cap C_{v_{i}}$ along the orientation. We also choose a point on each vanishing cycle $C_{v}$, which comes from the choice of the non-trivial spin structure. Then it follows from the Seidel's sign rule [29, Section (9e)] that the sign in (4) is given by $(-1)^{\dagger}$, where $\dagger$ is the sum of the number of $i \in[1, k]$ such that $\xi_{i}$ points away from the polygon and the the number of points on the boundary of the polygon coming from the spin structures.

Now we use the following fact from [10]: Let $G_{0}$ be the simplest dimer model consisting of one black node, one white node and three edges, which corresponds to the McKay quiver for the trivial group. Then any consistent dimer model can be obtained from $G_{0}$ by successively adding a divalent node or an edge.

Assume that a dimer model $G$ is obtained from another dimer model $G^{\prime}$ by adding an edge between a black node $b$ and a white node $w$ as shown in Figure 2. It follows 
from the definition of the grading of $M$ and $C_{v}$ that $\xi_{i}$ either points away from the polygon or toward the polygon simultaneously for all $i$, and the fact that $b$ and $w$ are adjacent in $G$ implies that if $\xi_{i}$ at $b$ points away from the disk corresponding to $b$, then $\xi_{i}$ at $w$ points toward the disk corresponding to $w$, and if $\xi_{i}$ at $b$ points toward the disk corresponding to $b$, then $\xi_{i}$ at $w$ points away from the disk corresponding to $w$. Then either of the disk at $b$ or $w$ receives an extra sign for $G$ compared to that for $G_{0}$, which can be off-set by the introduction of a new base point on the vanishing cycle, which is necessary since one of the vanishing cycles for $G^{\prime}$ is divided into two by adding an edge to $G^{\prime}$. This shows that the dimer model $G$ satisfies the assertion of the lemma if the dimer model $G^{\prime}$ does. Since the addition of a divalent node does not change $M$ and $C_{v}$ at all, the lemma is proved.

The double suspension $p^{\sigma \sigma}: \mathcal{S} \times \mathbb{C}^{2} \rightarrow \mathbb{C}$ of $p: \mathcal{S} \rightarrow \mathbb{C}$ is defined by

$$
p^{\sigma \sigma}(x, u, v)=p(x)+u v
$$

and a vanishing cycle $C \subset p(0)$ naturally gives a vanishing cycle $L \subset\left(p^{\sigma \sigma}\right)^{-1}(0)$ called the double suspension of $C$. The following theorem is due to Seidel:

Theorem 4.6 (Seidel [26, Corollary 5.5]). The full subcategory of $\mathfrak{F u k}\left(\left(p^{\sigma \sigma}\right)^{-1}(0)\right)$ consisting of double suspensions $L_{v}$ of $C_{v}$ for $v \in V$ is equivalent to the trivial extension of $\mathfrak{F u k} p$ of degree 3.

Since $\mathcal{A}$ is the trivial extension of $\mathcal{A}^{\rightarrow}$ of degree 3 (or the 3-dimensional cyclic completion in the terminology of [25]), Corollary 1.2 is proved.

\section{Acknowledgements}

We thank Bernhard Keller for several remarks which led to a major revision of this paper. We also thank Alastair Craw for pointing out the reference [4], Nils Carqueville and Alexander Quintero Velez for sending an earlier version of [4], and the anonymous referee for suggesting several improvements. K. U. thanks Akira Ishii for helpful discussions. M. F. is supported by Grant-in-Aid for Young Scientists (No.19.8083). K. U. is supported by Grant-in-Aid for Young Scientists (No.20740037). This work has been done while K. U. is visiting the University of Oxford, and he thanks the Mathematical Institute for hospitality and Engineering and Physical Sciences Research Council (EP/F055366/1) for financial support.

\section{References}

[1] A. I. Bondal and M. M. Kapranov, Enhanced triangulated categories, Mat. Sb. 181 (1990), no. 5, 669-683.

[2] T. Bridgeland, A. King, and M. Reid, The McKay correspondence as an equivalence of derived categories, J. Amer. Math. Soc. 14 (2001), no. 3, 535-554 (electronic).

[3] N. Broomhead, Dimer models and Calabi-Yau algebras. ArXiv:0901.4662.

[4] N. Carqueville and A. Quintero Velez, Remarks on quiver gauge theories from open topological string theory. ArXiv:0912.4699.

[5] B. Davison, Consistency conditions for brane tilings. ArXiv:0812.4185.

[6] B. Feng, Y.-H. He, K. D. Kennaway, and C. Vafa, Dimer models from mirror symmetry and quivering amoebae, Adv. Theor. Math. Phys. 12 (2008), no. 3, 489-545.

[7] V. Ginzburg, Calabi-Yau algebras (2006). Math.AG/0612139.

[8] V. K. A. M. Gugenheim, L. A. Lambe, and J. D. Stasheff, Perturbation theory in differential homological algebra. II, Illinois J. Math. 35 (1991), no. 3, 357-373. 
[9] A. Ishii and K. Ueda, Dimer models and exceptional collections. ArXiv:0911.4529.

[10] - Dimer models and the special McKay correspondence. ArXiv:0905.0059.

[11] _- On moduli spaces of quiver representations associated with dimer models, in Higher dimensional algebraic varieties and vector bundles, RIMS Kôkyûroku Bessatsu, B9, 127-141, Res. Inst. Math. Sci. (RIMS), Kyoto (2008).

[12] T. V. Kadeishvili, The algebraic structure in the homology of an $A(\infty)$-algebra, Soobshch. Akad. Nauk Gruzin. SSR 108 (1982), no. 2, 249-252 (1983).

[13] B. Keller, Koszul duality and coderived categories (after $K$. Lefèvre). Available at http://people.math.jussieu.fr/ keller/publ/.

[14] ——, A-infinity algebras in representation theory, in Representations of algebra. Vol. I, II, 74-86, Beijing Norm. Univ. Press, Beijing (2002).

[15] —_ Calabi-Yau triangulated categories, in Trends in representation theory of algebras and related topics, EMS Ser. Congr. Rep., 467-489, Eur. Math. Soc., Zürich (2008).

[16] K. D. Kennaway, Brane tilings, Internat. J. Modern Phys. A 22 (2007), no. 18, 2977-3038.

[17] A. D. King, Moduli of representations of finite-dimensional algebras, Quart. J. Math. Oxford Ser. (2) 45 (1994), no. 180, 515-530.

[18] M. Kontsevich, Homological algebra of mirror symmetry, in Proceedings of the International Congress of Mathematicians, Vol. 1, 2 (Zürich, 1994), 120-139, Birkhäuser, Basel (1995).

[19] — Lectures at ENS Paris, Spring 1998 (1998). Set of notes taken by J. Bellaiche, J.-F. Dat, I. Martin, G. Rachinet and H. Randriambololona.

[20] M. Kontsevich and Y. Soibelman, Stability structures, motivic Donaldson-Thomas invariants and cluster transformations. ArXiv:0811.2435.

[21] —_ Homological mirror symmetry and torus fibrations, in Symplectic geometry and mirror symmetry (Seoul, 2000), 203-263, World Sci. Publishing, River Edge, NJ (2001).

[22] K. Lefèvre-Hasegawa, Sur les A-infini catégories. ArXiv:math/0310337.

[23] S. A. Merkulov, Strong homotopy algebras of a Kähler manifold, Internat. Math. Res. Notices (1999), no. 3, 153-164.

[24] S. Mozgovoy and M. Reineke, On the noncommutative Donaldson-Thomas invariants arising from brane tilings. ArXiv:0809.0117.

[25] E. Segal, The $A_{\infty}$ deformation theory of a point and the derived categories of local Calabi-Yaus, J. Algebra 320 (2008), no. 8, 3232-3268.

[26] P. Seidel, Suspending Lefschetz fibrations, with an application to local mirror symmetry. ArXiv:0907.2063.

[27] — More about vanishing cycles and mutation, in Symplectic geometry and mirror symmetry (Seoul, 2000), 429-465, World Sci. Publishing, River Edge, NJ (2001).

[28] — Vanishing cycles and mutation, in European Congress of Mathematics, Vol. II (Barcelona, 2000), Vol. 202 of Progr. Math., 65-85, Birkhäuser, Basel (2001).

[29] _ Homological Mirror Symmetry for the quartic surface (2003). Math.AG/0310414.

[30] —_ Fukaya categories and Picard-Lefschetz theory, Zurich Lectures in Advanced Mathematics, European Mathematical Society (EMS), Zürich (2008), ISBN 978-3-03719-063-0.

[31] K. Ueda and M. Yamazaki, Homological mirror symmetry for toric orbifolds of toric del Pezzo surfaces. Math.AG/0703267.

[32] ——, A Note on Brane Tilings and McKay quivers. Math.AG/0605780.

[33] M. van den Bergh, Non-commutative crepant resolutions, in The legacy of Niels Henrik Abel, 749-770, Springer, Berlin (2004).

Graduate School of Mathematical Sciences, The University of Tokyo, 3-8-1 Komaba

MEgURO-KU TOKYO 153-8914, JAPAN

E-mail address: futaki@ms.u-tokyo.ac.jp

Department of Mathematics, Graduate School of Science, Osaka University,

Machikaneyama 1-1, Toyonaka, Osaka, 560-0043, JAPAN.

E-mail address: kazushi@math.sci.osaka-u.ac.jp 